\title{
VICTour 1.1: Introducing virtual learning environments and gamification
}

\author{
Curtis L. Maines ${ }^{1}$, Stephen Tang ${ }^{2}$ and David Llewellyn-Jones \\ Department of Computer Science \\ Liverpool John Moores University \\ Liverpool, L3 3AF, United Kingdom \\ Email: c.1.maines@2011.ljmu.ac.uk ${ }^{1}$, o.t.tang@1jmu.ac.uk ${ }^{2}$
}

\begin{abstract}
Game engines are one of the few solutions to providing a true virtual campus tour experience. In this paper, we explore the evolution of the virtual campus tour and thereby the current and future work of game technology within virtual touring. An investigation is conducted into the application of objective-based gamification and its ability to encourage exploration of a virtual world. We also examine virtual campus tours as an alternate form of taught content via virtual learning environments (VLEs). An investigation into the use of head mounted displays to improve immersion is also explored.
\end{abstract}

Index Terms-Interactive Virtual Campus Tour; Gamification; Virtual Learning Environment; Virtual Reality

\section{INTRODUCTION}

Virtual tours supply information via multimedia, giving users the impression they are navigating locations and experiencing them in real time [1]. Real virtual tours aim to provide an experience as close to visiting the actual location as possible. From a visual perspective this is accomplished by using characteristics which realistically represent the place, giving a uniform environment which makes the virtual and real locations appear somewhat familiar. These characteristics include exact imagery or representation of objects, accurate layout, inclusion of major land marks and facilities, interactivity and the ability to freely navigate in a virtual environment. To achieve full immersion an application would require satisfying all the senses. However, for this project the focus will be on vision.

The advancement of game technologies has made it possible to create realistic, highly interactive environments in an affordable way and with relatively quick production time [2]. In our previous paper [3], we investigated the use of game engines to develop a highly interactive virtual campus tour application. Our findings demonstrated that game technology is currently the only solution for providing a rich virtual tour experience. Meeting the requirements of realistic resemblance, accurate layout, interactivity and full exploratory freedom.

This paper discusses the next stage of our work, including an investigation into the use of objective-based gamification methods to encourage exploration and virtual reality to improve immersion. Section II describes the evolution of virtual campus tour solutions from those that are currently in use by most universities to our solution. Section III discusses other applications using game technology to create virtual worlds.
Section IV provides an overview on game technologies and the tools that support content creation. The implementation of the new features are then described in Section V. Finally, Section VI concludes and presents our future work in the area of virtual campus tours.

\section{Evolution of Virtual CAmpus Tour Solutions}

A virtual tour is an effective way to show the facilities of a location to the public. Many universities around the world have some form of virtual tour on their website to showcase their respective campuses. Based on our survey of fourteen universities, the types of virtual campus tour provided include: still image galleries, video galleries and $360^{\circ}$ interactive virtual tours.

\section{A. Still Image Galleries}

Still image galleries have become a lot scarcer since our previous paper, with many universities now opting for other solutions. Image galleries provide photographs of various locations throughout their respective campuses. However few universities exclusively use this solution and most run it alongside others. Universities which feature an application such as this include: Yale, Bangor, Nottingham and Bentley; see Fig. 1.

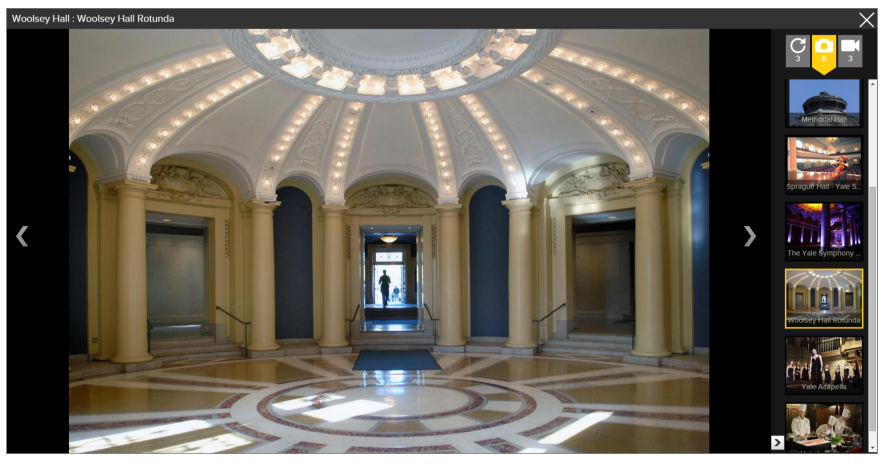

Fig. 1: Virtual Campus Tour of Yale University, Image Gallery.

The images in these galleries do not provide any form of information as to where they were taken within the campus. Their sole purpose is to present a positive impression of the buildings around the University. This kind of application is ambitious claiming itself as a virtual tour and is more acceptable for use in a promotional pamphlet. 


\section{B. Video Galleries}

Video galleries are effectively the evolution of image galleries, usually presented in a similar manner, but with far richer content. Videos provide continuous imagery, improving on still images and allowing for some attempt at a mental composition of a floor plan. The inclusion of sound can also supply an idea of the ambience of an area, providing additional detail of the real location.

Videos as a solution to virtual touring, however, do little more than images. Video-based virtual tours still provide very linear content and offer the user little in the way of control over what they view. They are also often accompanied by a presenter, inevitably blocking a large portion of the screen, thereby making any viewing of the surroundings less effective. The universities featuring video galleries include Dundee, Clark, Emory, New York, Middlesex, Yale, Bangor, Bentley and Nottingham Fig. 2.

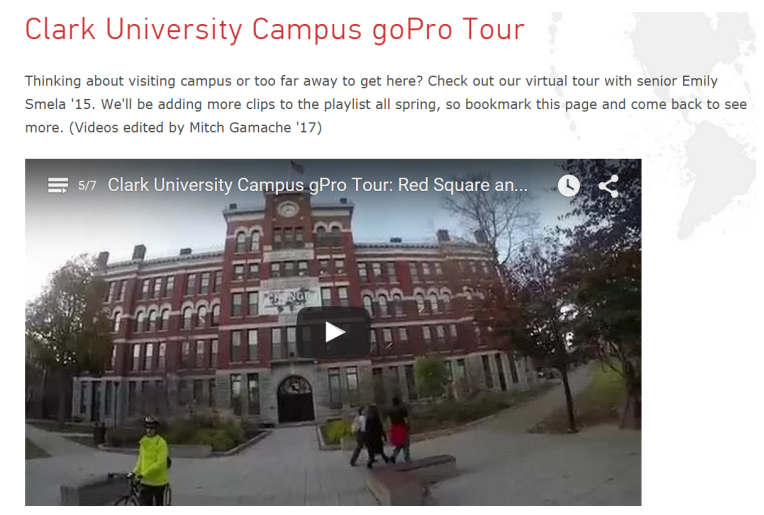

Fig. 2: Clark University, Video Gallery.

\section{C. $360^{\circ}$ Interactive Virtual Tours}

The $360^{\circ}$ interactive virtual tour is one of the few solutions which provides a real tour experience. There are two variants of this virtual tour solution: panoramic and $360^{\circ}$. Panoramic tours give the user the ability to view a room across the horizontal axis, scrolling left and right to view a continuous image around a location. Whereas the $360^{\circ}$ tours allow the user to move at any angle and have full control over what is viewed. Similar to that of a real world tour; allowing the user to choose what they view.

In comparison to an image gallery or video gallery, a $360^{\circ}$ tour provides an improved experience; allowing users to interact with the viewport as opposed to just observing it. This method however, is still not a perfect solution. Users are able to view locations from various angles but they are always viewing it from a fixed location. In addition, there are also limitations on where they can view and there is no indication of how areas are linked due to the excessive blind spots these tours suffer from. There are some applications such as Google Street View ${ }^{1}$ which provide very little blind spots, however

\footnotetext{
${ }^{1}$ Google, "University of Bedfordshire Virtual Tour - Google Street view," 2009, <http://www.beds.ac.uk/putteridgebury/virtual-tour-google-streetview $>$ (accessed 30/07/2015)
}

they still limit the user to where they can and can not explore. The universities which feature this type of tour include Sussex, Queen's, Kingston, San Diego State, Yale, Bangor, Bentley, Middlesex, Emory and Newcastle see Fig. 3.

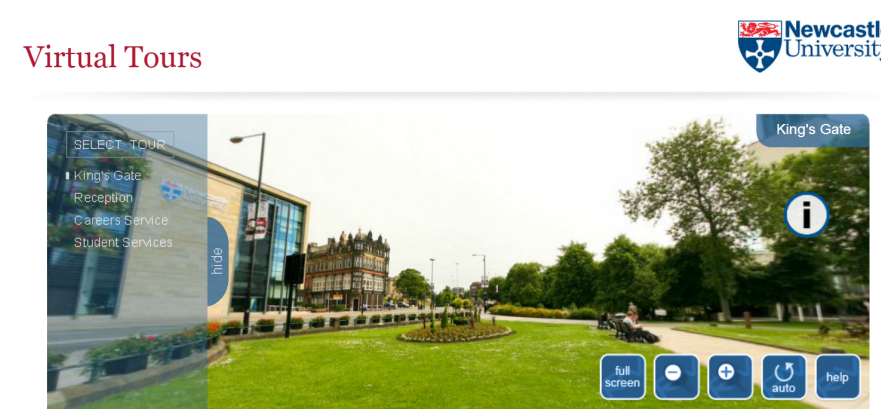

Fig. 3: Newcastle University, $360^{\circ}$ Interactive Virtual Tour.

\section{VICTour 1.0}

Our first solution, VICTour 1.0 (Virtual Interactive Campus Tour), solves all the aforementioned issues including: no location information, liner content, fixed locations, blind spots and poor interactivity. The user knows exactly what building and floor they're located on, and they have a map of the environment. They also have full exploratory freedom with no fixed locations or locked camera panning.

The viewport of VICTour 1.0 is configured to provide the user with a first person camera perspective. The user controls the angle of the camera using a mouse and WSAD (or arrow keys) to control movement within the virtual world. The minimap shown in Fig. 4 and Fig. 5 details the user's current location, including the building name and floor number.

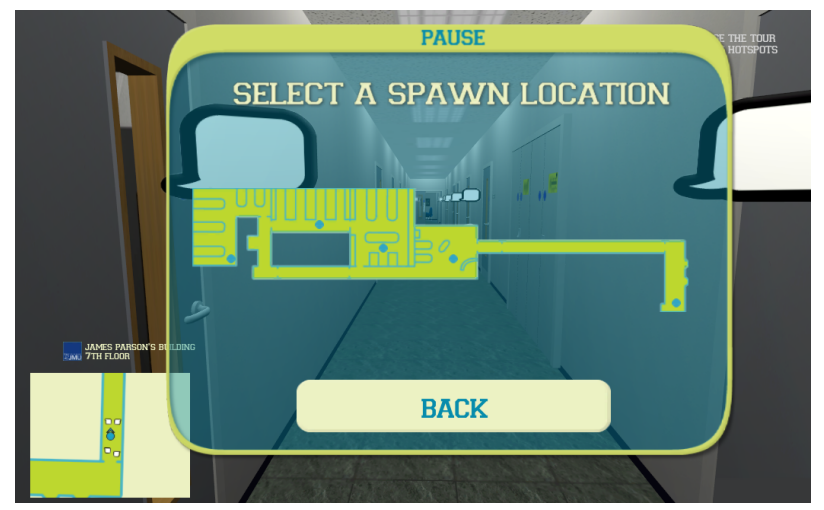

Fig. 4: Screenshot of spawning menu.

The map seen in Fig. 4 also provides the user with the ability to respawn at various points throughout the university. The first person camera is repositioned at the user's target location allowing them to explore the area they want.

The speech bubbles shown in Fig. 5 expand on collision. These provide textual information to the user about each section and room whilst they are exploring the area.

For less technology-savvy users, the automated tour also provides a linear virtual touring experience requiring little to no input. This is achieved through definition of paths for the camera to follow in each area. This mode of VICTour is less 


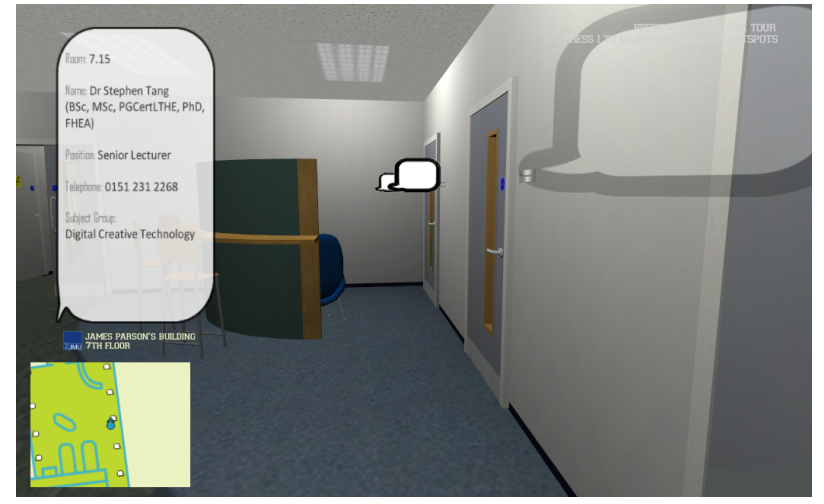

Fig. 5: Information pops up when user collides with speech bubbles.

interactive, limiting users to only select the previous or next area at each stage and pausing of the camera movement (see Fig. 6).

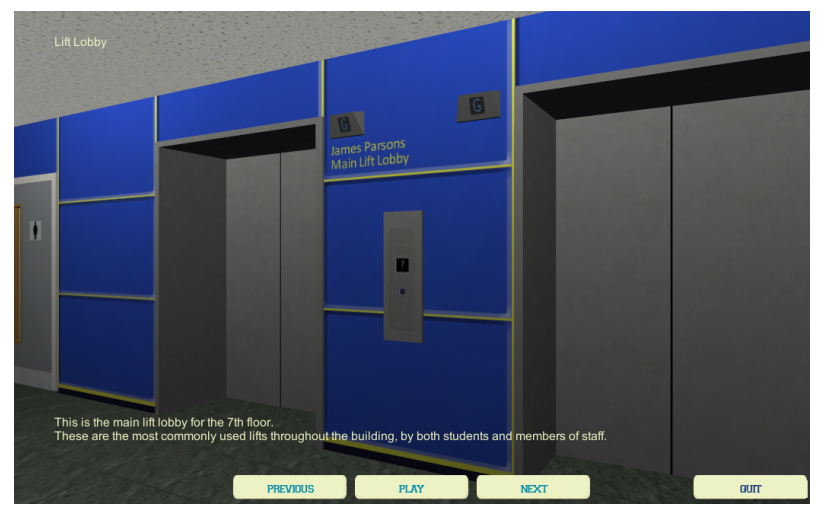

Fig. 6: Automated mode in VICTour 1.0.

\section{GAMES AND ViRTUAL WORLDS}

In this section, we survey other applications utilising game technology to create virtual environments. Including examples from both the entertainment and virtual worlds.

\section{A. Second Life}

Second Life [4] is a 3D world created by its player community. Every character is controlled by a real person and every object has been created by the users as opposed to the developers [5]. Some of the most popular uses for Second Life is the creation of a virtual environment for teaching, business and as a general virtual community. At its peak between 2005 and 2009, more than 300 universities worldwide were active users [6].

Although the pace of Second Life has somewhat slowed in recent years, many of its current applications align with our own for VICTour. One of our main goals is to implement the entire Liverpool John Moores University (LJMU) campus and incorporate VLEs such as those in Second Life.

A natural question that arises is why we chose not to implement our project in Second Life. This was mainly due to the rather poor graphic quality and cost associated with development in Second Life.

\section{B. Grand Theft Auto V}

Grand Theft Auto V (GTAV) [8], a somewhat controversial game [7] developed by Rockstar North, offers a very impressive open virtual world experience. At around $80 \mathrm{~km}^{2}$ in size, including a densely populated city with many shop interiors as well as a fully explorable ocean it is one of the better examples of a virtual world available in games today. Moderately based on the city of Los Angeles, GTAV also provides a partial virtual tour experience of its own.

GTAV is a strong example of the applications that can be created using game technology. This is particularly impressive when considering the target platform for the title was released in 2005.

Considerable inspiration was taken from this title, in particular the mini map in the lower left corner and the smartphone pop-up menu in the lower right (see Fig. 7). Mini maps are now a common appearance in open world games providing users with what is essentially a satellite navigation system of the game world. The smart phone idea was taken due to the target audience being undergraduate students and this being a particularly relatable device.

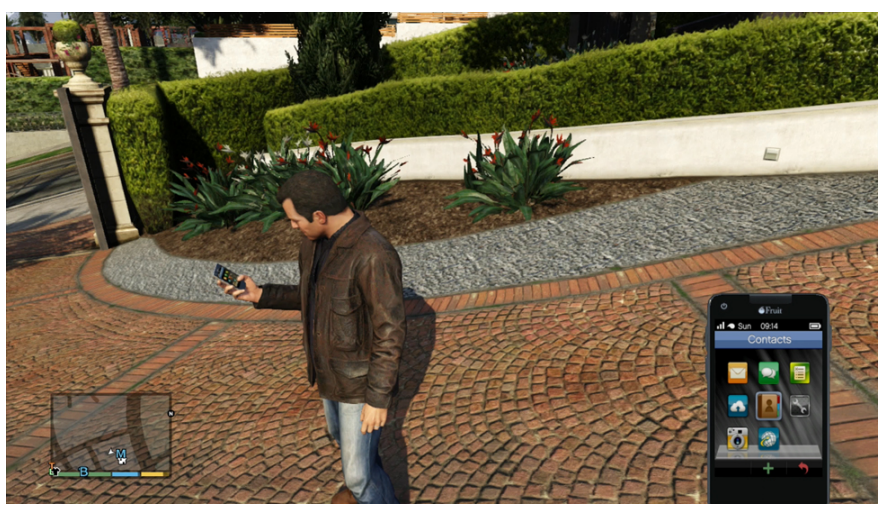

Fig. 7: Grand Theft Auto V Screenshot [8].

\section{Games Technology and Digital Content CREATION}

Modern 3D video games provide more than just an objective, enemy and set of rules. Games today provide highly accurate physics along with complex realistic environments [2]. The same technology can be used to create interactive virtual campus tours that allow users to freely explore the interior of a building modelled after the physical artefact.

In this section, we identify the key game technologies used for the creation of VICTour and describe the tools that are used to develop the 3D assets and compose the scene for the virtual campus tour.

\section{A. Software Requirements for the Development of VICTour}

Game engines provide the core technologies that aid developers in producing a variety of games rapidly. These components are used to handle tasks such as user input from human interface devices and Graphical User Interfaces (GUI), 
simulating motion, artificial behaviour and physicality in virtual environments, updating internal states of game objects and rendering visuals on the screen [9].

For the development of VICTour, the following game engine components are crucial to its operation.

- Renderer is responsible for displaying both 2D and 3D assets on the screen.

- Game Physics allow for the application of real world restrictions to the virtual world such as preventing the user from flying or travelling through walls.

- Lighting helps to illuminate and add more depth to the 3D objects within the virtual environment.

- Graphical User Interfacs and Head Up Displays (HUD) provides the necessary visual elements to display information and present graphical-based input options to the user.

- Inputs enable human interface devices such as keyboard and mouse to relay the user's action, which can then be mapped to actions that allows the user to explore the virtual environment.

- Scripting allows developers to access game engine functionality and program the mechanics and interaction of VICTour without the need to alter any of the game engine implementation.

\section{B. Game Level Editor}

In the production of computer games, game designers often rely on tools such as game level editors to help them author the virtual environment using assets that are produced using digital content creation tools [10]. Many features within the game engine can be turned on and off via the User Interface (UI) within the game level editor. This simplifies the workflow and reduces the total build time. Examples of game level editors include Unreal Engine ${ }^{2}$ and Unity Game Engine ${ }^{3}$.

\section{Digital Content Creation Tools}

Digital Content Creation (DCC) tools are used to produce all the assets such as 2D graphics, 3D models and audio for the purpose of virtual world scene composition. For 2D graphics, tools such as Adobe Photoshop are used to create the GUI and textures that will be used for the $3 \mathrm{D}$ models. The creation of $3 \mathrm{D}$ assets requires the use of $3 \mathrm{D}$ modelling tools such as $3 \mathrm{D}$ Studio Max and Maya. These tools also provide the facility to create key-framed 3D animation which can be ported to the game engine. Alternatively, 3D assets can be produced using digital sculpting tools such as Mudbox and ZBrush which provide more fluid manipulation of vertices to ease the creation of form and textures for organic-shaped models [11].

These tools are generally used by digital artists to create 2D or 3D artefacts for use in the population of virtual worlds. When creating assets they must conform to the requirements of the game engine otherwise they may be rendered incorrectly

\footnotetext{
${ }^{2}$ Epic Games, "Unreal Engine 4," 2014, <https://www.unrealengine.com > (accessed 30/07/2015)

${ }^{3}$ Unity Technologies, ”Unity 3D," 2015, <https://unity3d.com/> (accessed 30/07/2015)
}

or cause run-time performance issues. In the next section, we describe the process we undertook to create the new features for VICTour 1.1.

\section{VICTOUR 1.1: IMPLEMENTATION OF NEW FEATURES}

The beginning of our review in Section II identified the weaknesses of existing virtual campus tour solutions used by most universities to promote on-campus facilities. We have found that virtual campus tours built using game technologies can overcome the identified weaknesses and provide opportunities to incorporate interesting activities that further showcase services and facilities that are available to students and visitors.

\section{A. Scope and Requirements of VICTour 1.1}

Based on our assumption, the intended audience of the application are users aged between 18-50 years old. Users could be students, parents or guest lecturers to the University. These users seek information about facilities on offer as well as an accurate representation of the interior. All of which should be available off campus via the web.

LJMU operates across three separate campuses in the city of Liverpool. For VICTour 1.0 we limited the scope to cover only the 7th Floor of the James Parsons Building at the Byrom Street city campus. For VICTour 1.1 our aim has been to extend this to include an accurate representation of the 3rd Floor and James Parsons Upper Lecture theatre, including the open study areas, computer labs, research labs and staff offices. The 3rd floor is another part of the campus computing students will often find themselves, making it a necessity for VICTour 1.1. James Parsons Upper Lecture theatre is required to test VICTour's compatibility as a VLE and provide visitors with a virtual experience of a lecture in a University setting. This is to ensure that we give a positive impression and help users to visualise the environment through the use of the application. In order to achieve this, the layout of the site must resemble the physical world closely. The scale and aesthetic of the 3D objects needs to be realistic and clearly recognisable, and the virtual tour needs to include all the necessary objects such as chairs, desks and computers to populate the scene.

\section{B. Choice of Technologies}

There are two different clusters of technologies to decide on for the development of VICTour; (1) technology for producing the assets and (2) the game technology that runs the virtual campus tour.

For VICTour 1.0 and by extension 1.1, we have chosen to model the 3D assets using Autodesk 3DS Max and create the textures using Adobe Photoshop. Both of these tools provide the necessary functionalities to create $3 \mathrm{D}$ objects to compose the virtual environment.

Amongst the game technologies identified in Section IV.A, the Unity Game Engine was chosen for this project for two significant reasons. It provides a user friendly environment to develop a 3D game and it has well-documented online supports to aid developers. In addition, it allows games to be published to multiple platforms including the web via the Unity Web Player plugin. 


\section{Optimising Frame Rate and Performance}

Being web-based, VICTour will be accessible off campus. Therefore optimisation is a necessity to ensure all systems can run the application within the web browser smoothly. Left unoptimised, some users may be unable to load VICTour or suffer significantly lower frame rates. VICTour 1.0 was created around this constraint. Nevertheless, there were still some instances where the user would experience a drop in frame rate. Investigation found this to be due to an excessively high number of polygons being rendered needlessly. Fig. 8a. shows a wireframe view of VICTour 1.0. The red lines represent the boundary of the camera viewport and therefore what is rendered to screen. As seen there are large numbers of polygons being rendered outside of the camera's field of view which are blocked by other objects and could be removed. To overcome this issue, we used Unity's built in occlusion culling feature.

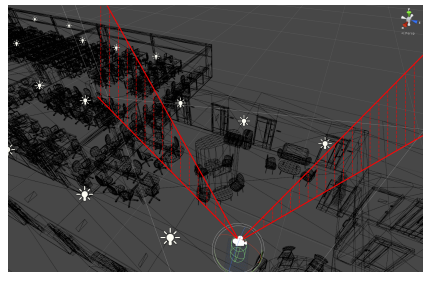

(a) Without occlusion culling

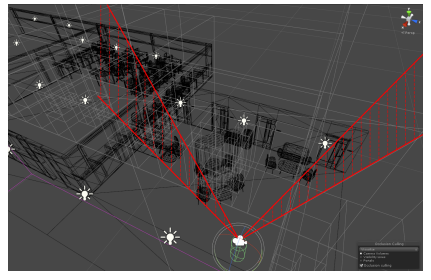

(b) With Occlusion Culling
Fig. 8: VICTour occlusion culling comparison

Unlike frustrum culling which only omits objects outside the camera's viewport, occlusion culling also disables objects which are blocked by other objects [12].The benefits of this feature can better understood by viewing Fig. $8 b$.

As seen the number of polygons now being rendered has dropped significantly. By enabling occlusion culling the frame rate for VICTour 1.1 was greatly increased by $36 \%$ on average and therefore improving the overall performance of the application.

\section{Improving Immersion with Oculus Rift VR}

Feedback from VICTour 1.0 supported our hypothesis that the use of game engines can provide users with a more realistic virtual tour experience [3]. Nevertheless, a typical desktop computer system still did not provide the level of immersion we felt could be achieved using today's technology.

In recent years, virtual reality (VR) head-mounted displays (HMD) have become more accessible than in the past. This is largely due to the launch of the Oculus Rift development kits [13]. These kits have given developers and academics alike access to technology which can enhance usability and user experience in domains such as game technology, education and medicine [14].

To improve the level of immersion users experience when using VICTour, we updated it to allow for Oculus Rift support (see Fig. 9). Fortunately, Unity Technologies had already created a plugin for Oculus Rift, making the incorporation of the technology a simple process.

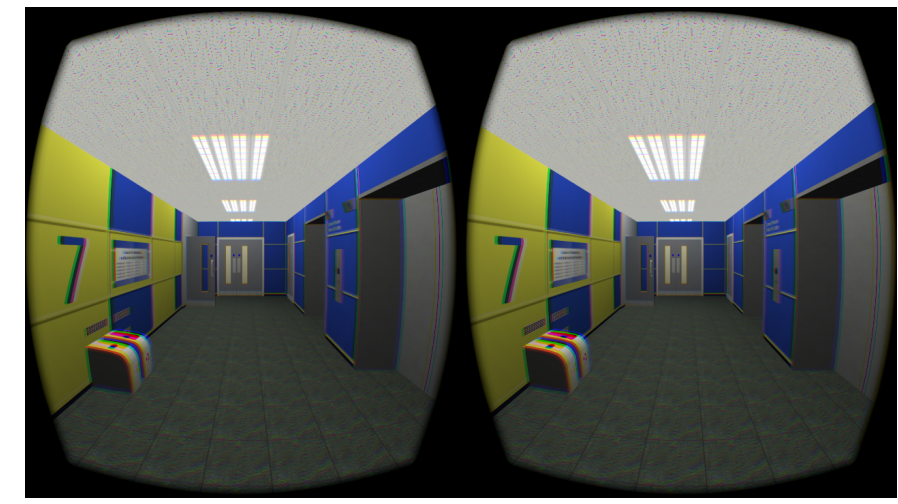

Fig. 9: VICTour 1.1 in virtual reality mode.

We have yet to carry out a comprehensive investigation with Oculus Rift. However, early experimentation indicates that users gain a much richer and more representative experience of visiting the campus when using the technology. Of the 12 participants who tested the application in pilot study 1, all responded that they felt more immersed using Oculus Rift and would like to visit other locations using the setup. In Section VI we discuss the future of Oculus Rift within our work and further approaches for testing this hypothesis.

\section{E. Incorporating Objectives for Gamification}

One theme that emerged from the feedback of VICTour 1.0, was the desire for some form of gamification. The challenge with implementing this suggestion is that we would be creating a game based on the campus, rather than a virtual tour of the campus. Typically, students, parents and visitors will be interested in a virtual tour of a university. A game is unlikely to be acknowledged given the competition it faces. However, after some deliberation we feel that we achieved a suitable balance between gamifying a virtual tour without turning it entirely into a game.

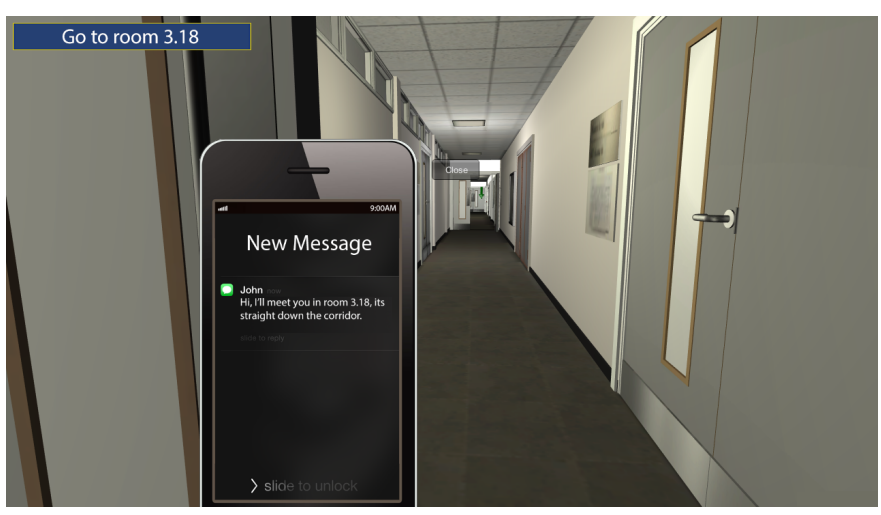

Fig. 10: VICTour 1.1 objectives.

This was done by creating objectives similar to those a typical student might carry out on a day-to-day basis. For example, the user would start in a corridor and be told to go to location $x$ to change their login credentials. This location is the same in the real world as in the virtual world. Thus providing the user (student) with the necessary knowledge of where in 
the building to go should they need to change their login details. These objectives were displayed via a smartphone in the application, see Fig. 10, an idea which was adapted from GTAV.

Of the 22 participants who tested VICTour 1.1's gamification module in pilot study 2, all of them found the objectives were easy to complete. $63.63 \%$ (14) of the participants found that completing the objectives assisted in building a cognitive map of the floor, while $27.27 \%$ (6) stated that it only provided minimal assistance. Overall, $72.72 \%$ (16) responded that the objectives definitely encouraged exploration.

\section{F. Expanding to include VLE}

Another application for VICTour was the creation of a VLE. VICTour 1.0 successfully showcased the campus and its facilities. However it didn't allow users to experience attending a lecture or lab session.

For VICTour 1.1, we developed a lecture theatre that included a playable video from the overhead projector. Given that the creation of several characters each with unique animations and audio would be a very lengthy process, for this purpose no characters were included. However, by playing the video the user is still able to get some perspective of how a university lecture differs from their college studies. The lecture theatre can be seen in Fig. 11.

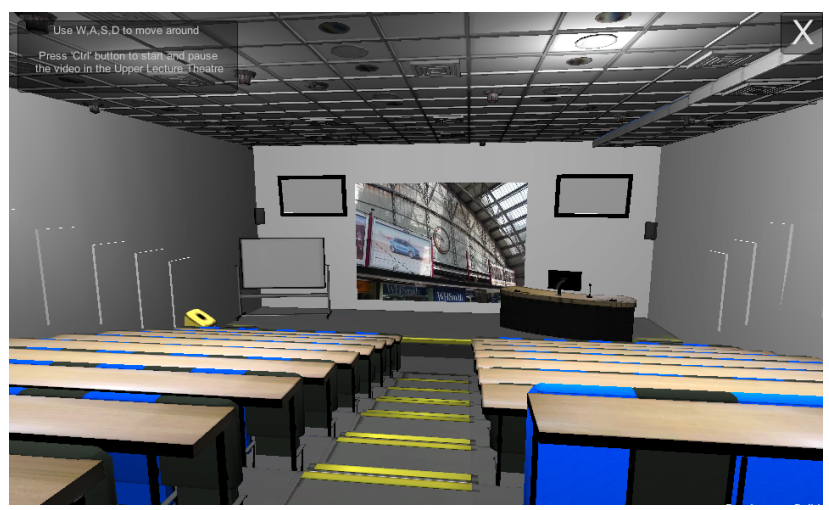

Fig. 11: VICTour 1.1 VLE; James Parsons Upper Lecture Theatre.

A group of students were asked to test VICTour 1.1's VLE (pilot study 3) and give their opinion on using it to view lectures or receive other content. Of the 12 surveyed, all expressed interest in receiving taught content this way. Some students however, did foresee potential issues with this method of teaching. The first being that lecturers may lose their passion if their only audience is a camera. They also fed back how at home they can become easily distracted and may lose interest in the lecture if viewing from a laptop rather than a lecture theatre. Overall, this method of teaching could be ran alongside traditional methods rather than as a replacement.

\section{CONCLUSIONS}

In this paper we surveyed the evolution of existing virtual campus tour solutions used by universities. VICTour 1.0 took advantage of game technology to provide users with a 3D immersive environment to freely explore from their web browser. VICTour 1.1 has taken the next step and begun to include VR HMD support, gamification and aspects of a virtual learning environment. Our pilot studies suggest there are benefits to using game technologies and gamification to improve prospective students' understanding of the university experience, and this therefore deserves further study. The immediate future of this project is to carry out a comprehensive investigation with Oculus Rift. Specifically around immersion levels experienced by the user when using a desktop setup versus Oculus Rift. We also aim to conduct experiments regarding location familiarity and whether or not Oculus Rift improves or hinders it. We will then return to gamification and VLEs to conduct a more thorough investigation into their impact on student learning.

\section{ACKNOWLEDEGMENT}

We would like to acknowledge Paul Humphreys and Darryl Cassidy, BSc (Hons) Computer Animation and Visualisation class of 2015 at LJMU for their involvement in the project. Paul implemented the gamification functionality and Darryl worked on the VLE within the virtual tour.

\section{REFERENCES}

[1] S. Bessey, Virtual 3D Environments: Implementations of 3D Environments for Virtual Tours and Online Communication, Thesis. Rochester Institute of Technology., Accessed from http://scholarworks.rit.edu/theses/6299, 2013.

[2] Y. Miche, M.-H. Lim, A. Lendasse and Y.-S. Ong, Meme representations for game agents, World Wide Web, Springer, vol. 18, no. 2, pp. 215-234, March 2015.

[3] C. Maines and S. Tang, An Application of Game Technology to Virtual University Campus Touring and Interior Navigation, in 7th International Conference on Development in e-Systems Engineering (DeSE2014), Phapos, 25th - 27th August, 2014.

[4] Second Life, Linden Research, Inc., [Online]. Available: http://www.secondlife.com/. [Accessed 14 July 2015].

[5] M. J. Jee, From First Life to Second Life: Evaluating taskbased language learning in a new environment, Canadian Journal of Learning and Technology, vol. 40, no. 1, pp. 1-15, 2014.

[6] F. Wang, J. K. Burton and J. Falls, A Three-Step Model for Designing Initial Second Life-Based Foreign Language Learning Activities, MERLOT Journal of Online Learning and Teaching, vol. 8, no. 4, pp. 324-333, December, 2012.

[7] P. M. Polasek, A Critical Race Review of Grand Theft Auto V, Humanity \& Society, vol. 38, no. 2, pp. 216-218, May 2014

[8] R. North, Grand Theft Auto V, Rockstar Games, September, 2013.

[9] S. Tang, M. Hanneghan and C. Carter, A Platform Independent Game Technology Model for Model Driven Serious Games Development, The Electronic Journal of e-Learning, pp. 61-79, 2013.

[10] E. Byrne, Game Level Design, Charles River Media, 2005.

[11] T. A. Galyean and J. F. Hughes, Sculpting: an interactive volumetric modeling technique, in 18th Annual Conference on Computer Graphics and Interactive Techniques, ACM, Ney York, NY, USA, 1991.

[12] U. Technologies, Unity Manual: Occlusion Culling, 10 July 2015. [Online]. Available: http://docs.unity3d.com/Manual/OcclusionCulling.html.

[13] S. Davis, K. Nesbitt and E. Nalivaiko, Comparing the onset of cybersickness using the Oculus Rift and two virtual roller coasters, in 11th Australasian Conference on Interactive Entertainment (IE 2015), Sydney, Australia, January, 2015.

[14] H. G. Hoffman, W. J. Meyer, M. Ramirez, L. Roberts, E. J. Seibel, B. Atzori, S. R. Sharar and D. R. Patterson, Feasibility of Articulated Arm Mounted Oculus Rift Virtual Reality Goggles for Adjunctive Pain Control During Occupational Therapy in Pediatric Burn Patients, Cyberpsychology, Behaviour, and Social Networking, vol. 17, no. 6, pp. 397-401, 2014. 